\title{
Performance Evaluation of Different Scheduling Schemes for Marcocell with MIMO Closed Loop Spatial Multiplexing (CLSM) 2×2 Antenna Configuration
}

\author{
Kyawt Kay Khaing \\ Department of Electronic \\ Engineering, \\ Technological University \\ Thanlyin, Myanmar
}

\author{
Zin Ma Ma Myo \\ Department of Electronic \\ Engineering, \\ Technological University \\ Thanlyin, Myanmar
}

\author{
Thae Hsu Thoung \\ Department of Electronic \\ Engineering, \\ Technological University \\ Thanlyin, Myanmar
}

\begin{abstract}
Nowadays, the usage of smart phones is very popular. More and more people access the Internet with their smart phones. This demands higher data rates from the mobile network operators. Every year the number of users and the amount of information is increasing dramatically. The wireless technology should ensure high data rates to be able to compete with the wire-based technology. The main advantage of the wireless system is the ability for user to be mobile. The 4G LTE system made it possible to gain very high peak data rates. The purpose of this paper was to investigate the improvement of the system performance for the downlink based on different antenna configurations and different scheduling algorithms. Moreover, the fairness between the users using different schedulers has been analyzed and evaluated.
\end{abstract}

Keywords: LTE, RR, PF, BCQI

\section{INTRODUCTION}

Long Term Evolution (LTE) is a broadband wireless access network offering a rich suite of multimedia applications such as $3 \mathrm{D}$ video conferencing, mobile $\mathrm{HD} \mathrm{TV}$ and real time streaming videos at very high vehicular speeds. These applications have heterogeneous Quality of Service (QoS) requirements such as priority, transmission delay, jitter, packet loss rate, packet error rate etc., to provide better user experience. QoS provisioning offers a challenge for the LTE network designers to efficiently utilize the limited available radio resources in a highly fading wireless medium. In order to meet this challenge, LTE standard incorporates Radio Resource Management (RRM) mechanisms such as Call Admission Control (CAC), scheduling etc., which are open issues for designers. Scheduling is a crucial RRM mechanism which divides and allocates radio resources among different users while maintaining QoS to optimize system performance.

The scheduling in both downlink and uplink is carried out by scheduler present at the Medium Access Control (MAC) sublayer of eNodeB (eNB). Since scheduling algorithm for eNB MAC scheduler is not standardized, LTE network designers have proposed scheduling algorithms which results in significantly different levels of user and system performance [3, 4].

LTE technology presents a very challenging multiuser problem: Several User Equipments (UEs) in the same geographic area require high data rates in a finite bandwidth with low latency. Multiple access techniques allow UEs to share the available bandwidth by allocating to each UE a fraction of the total system resources. The strong motivation beyond the resource allocation algorithms for scheduling is the improvement of system performance by increasing the spectral efficiency at the wireless interface and consequently enhancing the system capacity. Other constraints such as fairness must also be improved. Hence, it is important to find away to performance effective trade-off between efficiency and fairness. To develop an efficient scheduler to reach this trade-off, several factors must be taken into account such as: Signal-to-Interference-plus-Noise Ratio (SINR), packet delays, buffer status (queues length and packet delays), and type of service, fairness, channel conditions and complexity (time and computing) [2].

Hence in this paper an attempt has been made to evaluate the performance of Round Robin, Best CQI and Proportional Fair scheduling algorithms for Constant Bit Rate (CBR) traffic scenario using MATLAB based Vienna LTE Simulator.

\section{LTE MIMO (MULTIPLE INPUT MULTIPLE OUTPUT)}

The MIMO, Multiple Input Multiple Output is another of the LTE major technology innovations used to improve the performance of the system. This technology provides LTE with the ability to further improve its data throughput and spectral efficiency above that obtained by the use of OFDM. Although MIMO adds complexity to the system in terms of processing and the number of antennas required, it enables far high data rates to be achieved along with much improved spectral efficiency. As a result, MIMO has been included as an integral part of LTE.

\subsection{LTE MIMO Basics}

The basic concept of MIMO utilises the multipath signal propagation that is present in all terrestrial communications. Rather than providing interference, these paths can be used to advantage. 


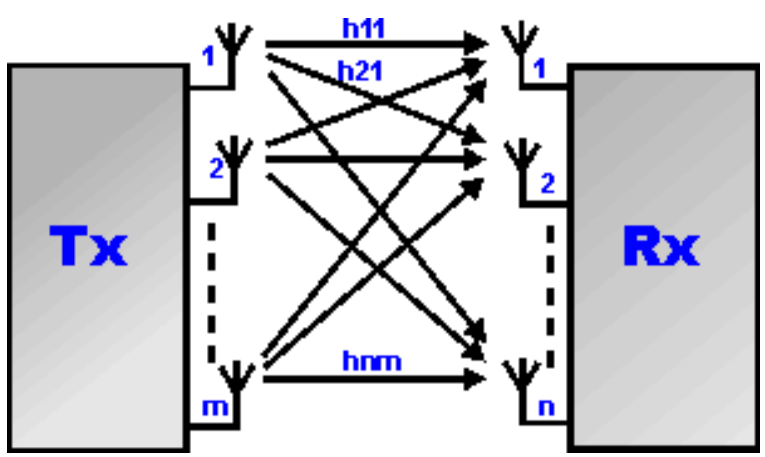

Figure 1. General Outline of MIMO system

The transmitter and receiver have more than one antenna and using the processing power available at either end of the link, they are able to utilise the different paths that exist between the two entities to provide improvements in data rate of signal to noise. MIMO is being used increasingly in many high data rate technologies including $\mathrm{Wi}-\mathrm{Fi}$ and other wireless and cellular technologies to provide improved levels of efficiency. Essentially MIMO employs multiple antennas on the receiver and transmitter to utilise the multi-path effects that always exist to transmit additional data, rather than causing interference. The use of MIMO technology has been introduced successively over the different releases of the LTE standards. MIMO has been a cornerstone of the LTE standard, but initially, in releases 8 and 9 multiple transmit antennas on the UE was not supported because in the interested of power reduction, only a single RF power amplifier was assumed to be available.

\section{LTE MIMO MODES}

There are several ways in which MIMO is implemented in LTE. These vary according to the equipment used, the channel function and the equipment involved in the link.

\subsection{Single Antenna}

This is the form of wireless transmission used on most basic wireless links. A single data stream is transmitted on one antenna and received by one or more antennas. It may also be referred to as SISO: Single In Single Out or SIMO Single In Multiple Out dependent upon the antennas used. SIMO is also called receive diversity.

\subsection{Transmit Diversity}

This form of LTE MIMO scheme utilises the transmission of the same information stream from multiple antennas. LTE supports two or four for this technique.. The information is coded differently using Space Frequency Block Codes. This mode provides an improvement in signal quality at reception and does not improve the data rate. Accordingly this form of LTE MIMO is used on the Common Channels as well as the Control and Broadcast channels.

\subsection{Open Loop Spatial Multiplexing}

This form of MIMO used within the LTE system involves sending two information streams which can be transmitted over two or more antennas. However there is no feedback from the UE although a TRI, Transmit Rank Indicator transmitted from the UE can be used by the base station to determine the number of spatial layers.

\subsection{Close Loop Spatial Multiplexing}

This form of LTE MIMO is similar to the open loop version, but as the name indicates it has feedback incorporated to close the loop. A PMI, Pre-coding Matrix Indicator is fed back from the UE to the base station. This enables the transmitter to precode the data to optimise the transmission and enable the receiver to more easily separate the different data streams.

\subsection{Closed Loop with Pre-Coding}

This is another form of LTE MIMO, but where a single code word is transmitted over a single spatial layer. This can be used as a fall-back mode for closed loop spatial multiplexing and it may also be associated with beamforming as well.

\subsection{Multi-User MIMO, MU-MIMO}

This form of LTE MIMO enables the system to target different spatial streams to different users.

\subsection{Beam-forming}

This is the most complex of the MIMO modes and it is likely to use linear arrays that will enable the antenna to focus on a particular area. This will reduce interference, and increase capacity as the particular UE will have a beam formed in their particular direction. In this a single code word is transmitted over a single spatial layer. A dedicated reference signal is used for an additional port. The terminal estimates the channel quality from the common reference signals on the antenna.

\section{LTE DOWNLINK SCHEDULING}

The air interface of LTE technology is based on OFDMA and SC-FDMA in the downlink and uplink respectively to deliver the flexibility and increase data rate without additional bandwidth or increase transmit power. The base station (eNodeB) is the entity responsible for controlling the air interface between the network and user equipments. The data transmission in LTE system is organized as physical resources which are represented by a time-frequency resource grid consisting of Resources Blocks (RBs) which has a duration of $0.5 \mathrm{~ms}$ and a bandwidth of $180 \mathrm{kHz}$ (12 subcarriers spaced with $15 \mathrm{kHz}$ ). It is a straight forward to see that each RB has $12 \times 7=84$ resource elements in the case of normal cyclic prefix and $12 \times 6=72$ resource elements in the case of extended cyclic prefix [8].

The scheduler entity have a role to assigns resources blocks every TTI, based on the channel condition feedback received from User Equipment in the form of Channel Quality Indicator (CQI) send by the UEs to the eNodeB, to indicate the data rate supported by the downlink channel. Every value of CQI, index in the range 1 to 15 , corresponds to the highest Modulation and Coding Scheme (MCS) and the amount of redundancy included [2].

In LTE system, the resource allocation is done in time and frequency domain. In time domain, the downlink channel is divided into frame of $10 \mathrm{~ms}$ each consists of 10 subframes of 1 ms each referred to as Transmission Time Interval (TTI). In frequency domain, the available system bandwidth is divided into sub-channels of $180 \mathrm{kHz}$, comprising of 12 consecutive equally spaced OFDM subcarriers of $15 \mathrm{kHz}$ each. A timefrequency radio resource spanning over $0.5 \mathrm{~ms}$ slots in the time domain and over $180 \mathrm{kHz}$ sub-channel in the frequency domain is called Resource Block (RB). The number of resource blocks in the available bandwidth is called Resource grid. Resource Element (RE) represents one OFDM subcarrier during one OFDM symbol interval. The number of RBs in a resource grid depends on the size of the bandwidth. The LTE operates in the bandwidth of $1.4 \mathrm{MHz}$ up to $20 \mathrm{MHz}$, with number of RBs ranging from 6 to 100 respectively. 
The scheduler at eNodeB attempts for appropriate allotment of the resources among UEs. The UE reports CQI (Channel Quality Indicator) which helps eNodeB to approximate the downlink channel quality. By the use of CQI report about the whole downlink bandwidth or about information about subband, the eNodeB can organize. CQI reporting for different sub-bands needs more uplink resources [6].

The channel dependent scheduling requires maintain-ing some fairness among the users and on the other hand it would like to provide higher cell throughput in their resource allocations. There is a connection between fairness and cell throughput.

Different scheduling methods are shown below in order to address this trade off [7].

\section{PERFORMANCE EVALUATION OF DIFFERENTSCHEDULING SCHEMES FOR MACROCELL}

With Vienna LTE Simulator being the simulator of interest, in order to demonstrate how effective simulator is, the following tasks are undertaken and how efficiently and easily the simulator reproduces the results that making it an effective simulator is shown. The first task is performance evaluation of various scheduling schemes with the evolution of the network.

In this paper, the performance of different scheduling schemes is compared and evaluated using the Vienna LTE Simulator. This study helps us to understand how the resources are allocated to each user and the various factors that affect the scheduling. This study also helps the service provider to offer reliable service at lower implementation cost and deploy a network that has ability to sustain the evolution.

\section{Simulation Results and Discussion}

The role of the scheduling schemes is to allocate the resource blocks to users depending on signal quality and channel conditions. The three well known scheduling schemes are Round Robin, Best CQI and Proportional Fair. In this section, these scheduling schemes are compared for their performance evaluation with two transmitting and two receiving antennas, and MIMO transmit mode is CLSM. The results obtained help us to decide the best scheduling scheme for a network to overcome network congestion and highlight features of the Vienna LTE simulator.vThe network under consideration has the properties as shown in Table 1.

TABLE 1. NeTWORK CONFIgURATION FOR COMPARING SCHEDULING SCHEMES

\begin{tabular}{|l|l|}
\hline System Bandwidth & $20 \mathrm{MHz}[50 \mathrm{RBs}]$ \\
\hline System Frequency & $2.14 \mathrm{GHz}$ \\
\hline $\begin{array}{l}\text { Number of } \\
\text { Transmitter }\end{array}$ & 2 \\
\hline Number of Receiver & 2 \\
\hline Number of Users & 20 Users per Cell \\
\hline Scheduling Algorithm & $\begin{array}{l}\text { Round Robin, Best CQI, } \\
\text { Proportional Fair }\end{array}$ \\
\hline Channel Model & Winner II \\
\hline Pathloss Model & TS36942 - Urban \\
\hline
\end{tabular}

The network is configured with the above mentioned parameters and the simulation is run on the Vienna LTE simulator. The network configuration is specified in the launcher file and few other parameters are specified in the simulation config file. The resulting fairness, edge user throughput, average user throughput and cell throughout are compared for each scheduling scheme starting from the Round Robin scheduling scheme. Figure 2 shows 20 randomly distributed users in a cell using Round Robin scheduling scheme.

.The users are distributed in such a way that most of them have good channel conditions. The users which are very close to base station are avoided because these users always have very good channel conditions and on using channel aware scheduling schemes most of resource blocks are allocated to these users, neglecting the users with lower CQI. To make a valid comparison between the channel aware and channel unaware scheduling algorithms, Users that are distributed in the region between the cell center and the cell edge are considered. The users are randomly distributed in the region of interest.

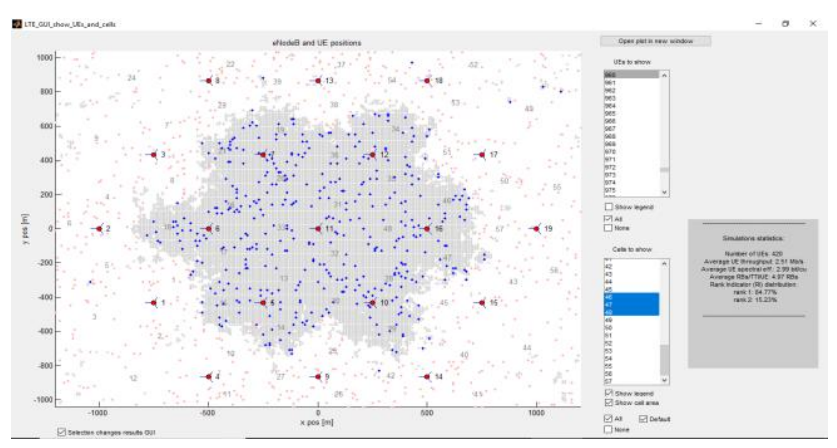

Figure 2. Network under Study of Round Robin Scheduling with 20 Users per Cell

For this network, simulation is run by attaching each user to its corresponding sector and each sector using the Round Robin scheduling scheme. Since Round Robin allocates the resources to each user requesting the service the fairness of this scheduling scheme is high and the results show the same. For simulation purposes, the traffic model is assumed to be full buffer, meaning that all the users have a packet to send. Figure 4.2 shows the simulation results for this setup run for 50 TTI's [Transmission Time Interval].

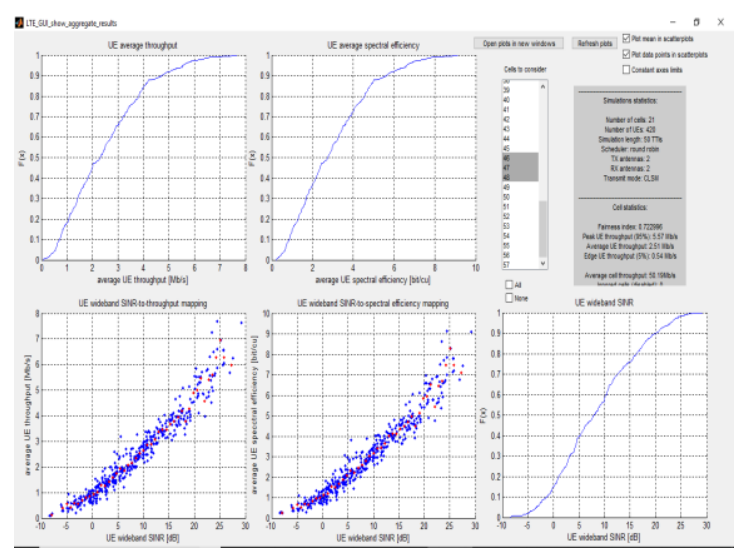

Figure 3. Simulation Results for Round Robin Scheme with 20 Users per Cell 
Figure 3 shows the various results that help in assessing the network operation based on network configuration. They show how the average throughput varies with the SINR and the spectral efficiency. The upper right corner displays the fairness index, average/peak and edge user throughput and also the total cell throughput. This network shows that the fairness index of the network is 0.72 which is as expected for the Round Robin as it serves all the users requesting service. Each user has average user throughput of $2.51 \mathrm{Mbps}$. The similar simulations are carried out for the Best CQI and Proportional Fair scheduling scheme and results are compared to evaluate best scheduling scheme under different network conditions with MIMO Closed Loop Spatial Multiplexing (CLSM) $2 \times 2$ antenna configuration.

In Figure 4 to Figure 7, simulation is run by attaching each user to its corresponding sector and each sector using the Best CQI and Proportional Fair scheduling schemes.

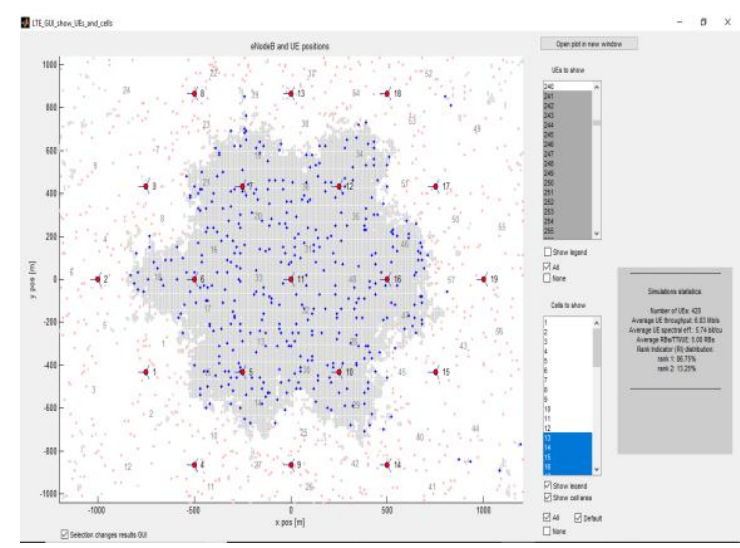

Figure 4. Network under Study of Best CQI Scheduling with 20 Users per Cell

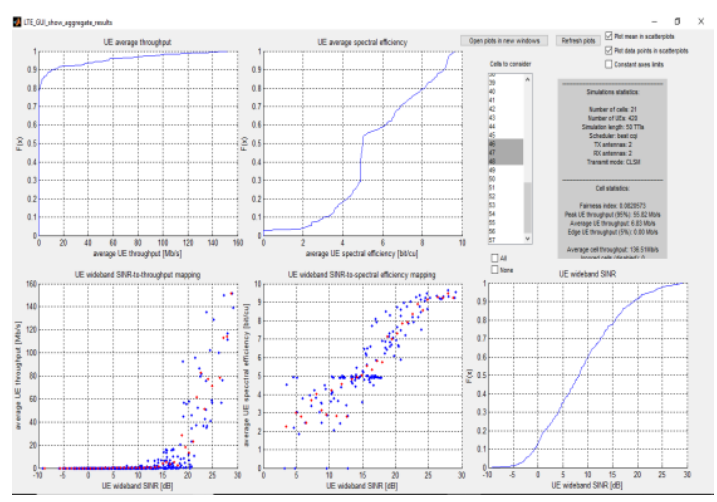

Figure 5. Simulation Results for Best CQI Scheme with 20 Users per Cell

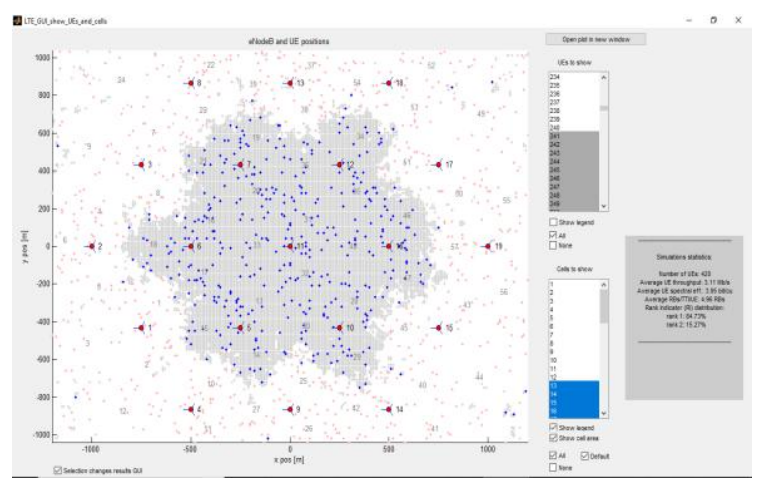

Figure 6. Network under Study of Proportional Fair Scheduling with 20 Users per Cell

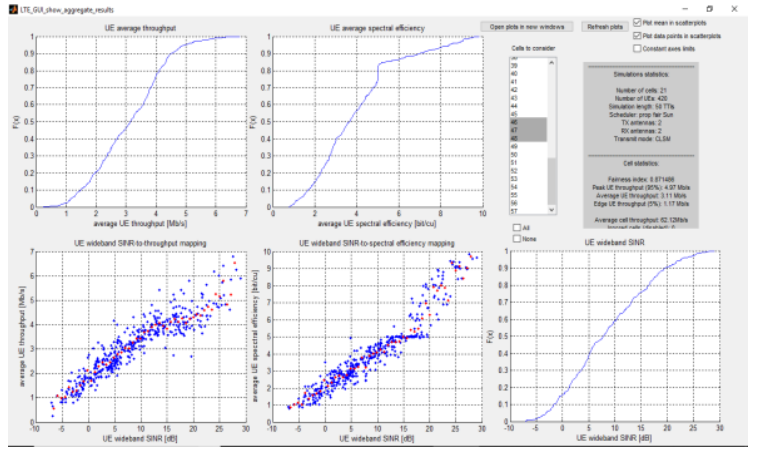

Figure 7. Simulation Results for Proportional Fair Scheme with 20 Users per Cell

The simulations were made with the LTE System Level Simulator 1.6. The LTE downlink OFDMA scenario is considered in the following simulations. Figure 8 illustrates the mean, edge and peak throughput.

Three different scheduling algorithms were chosen: Round Robin, Best Channel Quality Indicator and Proportional Fair. Closed Loop Spatial Multiplexing (CLSM) with $2 \times 2$ antenna configuration, $20 \mathrm{MHz}$ bandwidth and 20 users in the cell. The simulation time is chosen equal to 50 TTI where TTI is equal to $1 \mathrm{~ms}$. The pathloss model was chosen according to the 3GPP technical specification TS36.942. The scenario in the following simulation is done for the urban area model. The propagation model is the following:

$$
\begin{gathered}
\mathrm{L}=40\left(1-4.10^{-3} \cdot \mathrm{Dhb}\right) . \log _{10}(\mathrm{R})-18 . \log _{10}(\mathrm{Dhb})+21 . \\
\log _{10}(\mathrm{f})+80 \mathrm{~dB}
\end{gathered}
$$

where Dhb is the height of the base station antenna measured in metres from the average rooftop level, $\mathrm{R}$ is the base station UE separation in $\mathrm{km}$, and $\mathrm{f}$ is the carrier frequency in $\mathrm{MHz}$.

The carrier frequency was taken equal to the $2000 \mathrm{MHz}$ and $\mathrm{Dhb}=15 \mathrm{~m}$ above the average rooftop level according to the 3GPP technical specification TS36.942. Then the equation 10 will be in the following form:

$$
\mathrm{L}=128.1+37.6 \log _{10} \mathrm{R}
$$

After L propagation factor is found the pathloss can be described by the following formula:

$$
\text { Pathloss }_{\text {macro }}=\mathrm{L}+\log \mathrm{F}
$$

where $\operatorname{LogF}$ is the log-normally distributed shadowing with the standard deviation of $10 \mathrm{~dB}$.

For the first mean throughput it can be seen that the Best CQI has the maximum value and the Round Robin has the smallest one. The drawback of the Best CQI scheduler is a very low fairness among the users. It is happening because this scheduler gives the resources only to the user with the best channel conditions. The users that have all the time a bad channel quality will be not scheduled at all. In contrast, the Proportional Fair scheduler has the best fairness among the users. Despite on the very high peak throughput of Best CQI scheduler it is not serving the users that have a bad channel condition, the edge throughput is equal to 0 . 


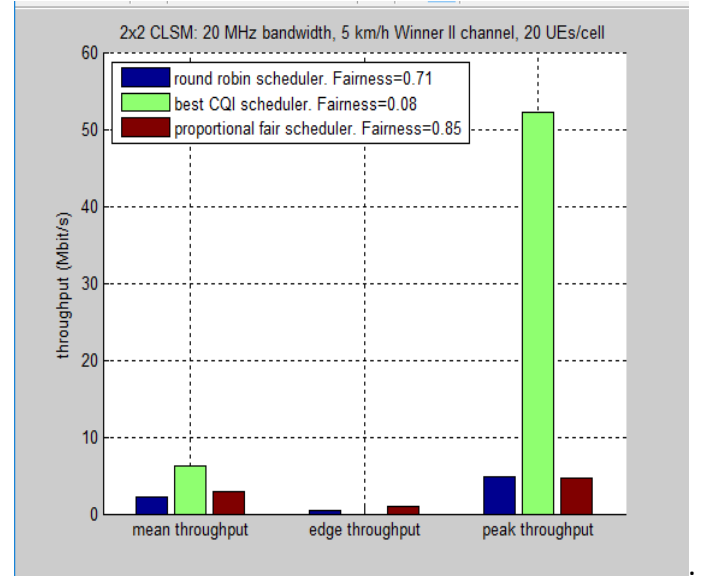

Figure 8. UE Throughput Comparison for Different Scheduling Algorithms

Figure 9 illustrates the empirical $\mathrm{CDF}$ of throughput of different scheduling algorithms. It can be seen that the probability of throughput to be equal to 0 for best CQI scheduler has the very big chances, the ECDF of it is about 0.64. But from the other side it can achieve the higher throughput than Proportional fair and Round Robin schedulers. The black dots on the graphs correspond to the mean value.

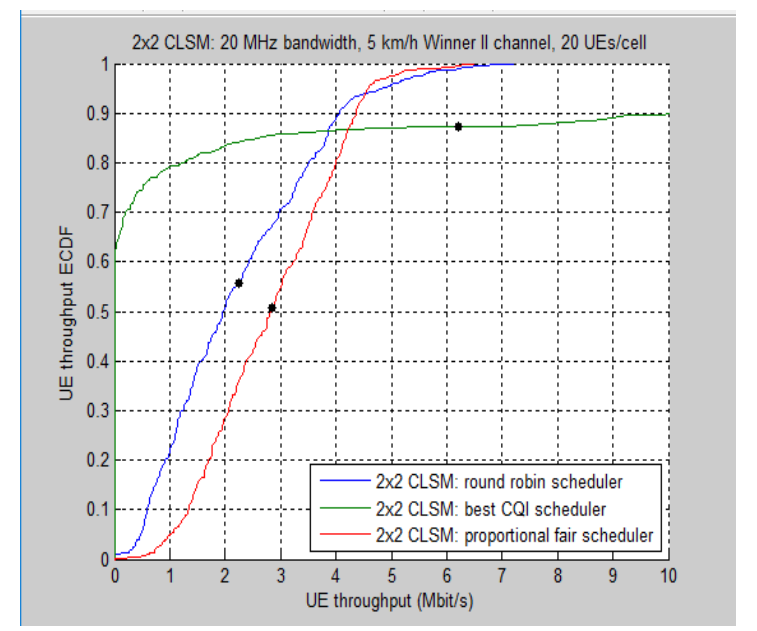

Figure 9. The Empirical CDF of the Throughput of Different Scheduling Algorithms

\section{CONCLUSION}

Long Term Evolution is the Fourth Generation that was developed in the 3GPP project. Long Term Evolution has improved the performance of the wireless communication significantly. It has the ability to achieve very high data rates using new technologies as OFDMA and MIMO.

The different scheduling algorithms of the downlink are simulated in this work. The Vienna LTE simulator which contains the LTE System Level Simulator is used. The results that were achieved demonstrate the multiple ways of how to improve the system performance.

The following results were collected after the simulation of different schemes:

The scheduling algorithm can improve the system performance. The right allocation of radio resources could increase the throughput of the system. Furthermore, the fairness between users has to be taken into account. According to the simulation results the best scheduler between Round Robin, Proportional Fair and Max CQI in respect to the fairness, became the Proportional Fair algorithm. Though, the Max CQI gave the highest peak data rate.

The increased number of transmitter and receiver antenna made the system get a higher data rate than SISO would provide.

\section{ACKNOWLEDGMENTS}

The author would like to express her profound gratitude to Dr. Theingi, Rector, Technological University (Thanlyin), for her encouragement and managements and the author would like to express her thanks to thesis supervisor, Dr. Zin Ma Ma Myo, Professor and Head of Department of Electronic Engineering, for her motivation and encouragement to complete this research in time. After all, the author would like to express her thanks to all her teachers and her parents, for their supports and encouragements.

\section{REFERENCES}

[1]. 3GPP TS 36.212 Version 10.0.0 Release 10, Evolved Universal Terrestrial Radio Access (E-UTRA): Multiplexing and channel coding, March, 2009.

[2]. M. AMMAR, B. NSIRI, W. HAKIMI and M. ELJAMAI, "A comprehensive study of Open-loop Spatial Multiplexing and Transmit Diversity for Downlink LTE”, International Journal of Computer Sciences and Telecommunication (IJCST), volume 5 Issue 2, January 2014.

[3]. Technical White paper: "Long Term Evolution (LTE): A Technical Overview," by Motorola.

[4]. S. M. Chadchan, "A Fair Downlink Scheduling Algorithm for 3GPP LTE Networks", I. J. Computer Network and Information Security, 6, pp 34-41, 2013. [6] D. Talevski and Li. Gavrilovska, "Novel Scheduling Algorithms for LTE Downlink Transmission", Telfor Journal, Vol. 4, No. 1, February 2012.

[5]. Dinesh Mannani, "Modeling and Simulation of Scheduling Algorithms in LTE Networks", Bachelor of Science Thesis report, Warsaw, January 2012.

[6]. Al. Bahillo Martinez, "Evaluation of multiuser scheduling algorithm in OFDM for different services", Master of Science in Electronics report, June 2006.

[7]. H. Seo and B. Gi Lee, "A Proportional Fair Power Allocation for Fair and Efficient Multiuser OFDM Systems", School of Electrical Engineering, Seoul National University, April 2004.

[8]. E. L. Hahne, "Round-Robin Scheduling and Window Flow Control for Max-Min Fairness in Data Networks" IEEE, technical report, November 1987.

[9]. Raymond H. Y. Louie, "Open-Loop Spatial Multiplexing and Diversity Communications in Ad Hoc Networks", Revised version. Submitted to IEEE Tr. Info Theory, 17 september 2010. 